Technological University Dublin

DƯBLIN

ARROW@TU Dublin

2010

\title{
A Computational Analysis of Cognitive Effort
}

Luca Longo

Technological University Dublin, luca.longo@tudublin.ie

Stephen Barrett

Trinity College Dublin, Ireland

Follow this and additional works at: https://arrow.tudublin.ie/scschcombk

Part of the Computational Engineering Commons, and the Computer Sciences Commons

\section{Recommended Citation}

Longo. L. \& Barrett, S. (2010). A computational analysis of cognitive effort. Intelligent Information and Database Systems, pg. 65-74. doi:10.1007/978-3-642-12101-2_8

This Book Chapter is brought to you for free and open access by the School of Computer Sciences at ARROW@TU Dublin. It has been accepted for inclusion in Books/Book Chapters by an authorized administrator of ARROW@TU Dublin. For more information, please contact arrow.admin@tudublin.ie, aisling.coyne@tudublin.ie,gerard.connolly@tudublin.ie.

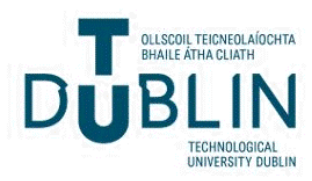




\title{
A Computational Analysis of Cognitive Effort
}

\author{
Luca Longo and Stephen Barrett \\ Department of Computer Science and Statistics - Trinity College Dublin \\ \{llongo, stephen.barrett\}@cs.tcd.ie
}

\begin{abstract}
Cognitive effort is a concept of unquestionable utility in understanding human behaviour. However, cognitive effort has been defined in several ways in literature and in everyday life, suffering from a partial understanding. It is common to say "Pay more attention in studying that subject" or "How much effort did you spend in resolving that task?", but what does it really mean? This contribution tries to clarify the concept of cognitive effort, by introducing its main influencing factors and by presenting a formalism which provides us with a tool for precise discussion. The formalism is implementable as a computational concept and can therefore be embedded in an artificial agent and tested experimentally. Its applicability in the domain of AI is raised and the formalism provides a step towards a proper understanding and definition of human cognitive effort.
\end{abstract}

Keywords: Cognitive Effort, Artificial Intelligence, Virtual Agents.

\section{Introduction}

Attention plays a central role in the behaviour of human beings. The concept of attention has been inconclusively studied in the history of psychology since the early years of the nineteen century. A plethora of definitions has been proposed and a large number of studies have been carried out in different directions. In 1908 Titchener 1 asserted that: "the doctrine of attention is the nerve of the whole psychological system, and that as men judge of it, so shall they be judged before the general tribunal of psychology". Behaviourists and Gestalt theories shared the conviction that the operations which relate output, such as response or percept, to input, such as stimulus or field, conform to a simple set of rules, such as isomorphism or conditioning [6]. By the end of the 1950s, the situation radically changed and the new concept of attention was a central topic in an emergent cognitive psychology that ascribed more spontaneity and autonomy to the organism implying some degree of local unpredictability than the previous classical doctrines. Post-behaviouristic psychology used the label of attention to denote some of the internal mechanisms that determine the significance of stimuli and therefore make it impossible to predict behaviour by stimulus consideration alone. In everyday language, attention is the act or faculty of attending, by directing the mind to an object or thought. Psychologists refer to attention as a state of consciousness characterised by such concentration.

N.T. Nguyen, M.T. Le, and J. Świątek (Eds.): ACIIDS 2010, Part II, LNAI 5991, pp. 65-74, 2010. (C) Springer-Verlag Berlin Heidelberg 2010 
Let's now consider the example of Luca, a young schoolboy. Luca does not like school that much, as most of his coetaneous, and for this reason he enjoys a pleasant state of drowsiness most of the time. When the teacher calls attention to him, Luca does not merely fail to pay attention but he has less attention to pay. This facts suggests that the drowsy schoolboy merely suffers from, or perhaps enjoys, a general low level of attention paying a small amount of cognitive effort. This example illustrates the dynamic construct of the concept of effort that changes within individuals in response to individual and environmental factors. This thesis is sustained by motivation theories [2 [3] and contrasts with recent empirical studies that have tended to treat effort as a static concept 4 . Berlyne suggested, in 1960, that the intensity of attention is related to the level of arousal that can be measured with electrophysiological techniques, and that is largely controlled by the properties of the stimuli to which the organism is exposed [5]. He was mainly concerned with involuntary attention. In voluntary attention, the subject attends to stimuli because they are relevant to a task that he has chosen to perform and not because of their arousing quality. This suggests that the intensive aspect of attention corresponds to effort rather than to mere wakefulness. Theories of information processing consider cognitive effort as a hypothetical construct, regarded as a limited capacity resources that affects the speed of information processing [6. In the work of Norman and Bobrow [7], if a task is resource-limited, then the performance will improve if more cognitive effort is allocated to the task. Although cognitive effort may be a hypothetical construct, it is a subjective state that people have introspective access to [8]. In the literature there are several attempts towards the measurement of cognitive efforts. It is a multi-faceted phenomenon: it can be related to physiological states of stress and effort, to subjective experiences of stress, mental effort, time pressure, and to objective measures of performance levels. These various aspects of cognitive effort have led to distinct means for assessing workload including physiological criteria such as heart rate, skin temperature, pupils dilation, blood pressure, respiration, performance criteria such as quantity and quality of performance by using primary task and secondary task measures, and subjective criteria such as rating of level of effort, self-report measures 9 . Despite an extensive literature, there appears to be no attempt to formalise the concept of cognitive effort as a computational concept therefore our goal is to begin the development of a formalism suitable for computations. Our research question here is:

\section{How can we formalise cognitive effort as a computational concept?}

We propose to develop a formalism, suitable for ongoing refinement, that captures the core aspects of a more complete theory. The subjective nature of the concept is noted in section 2 where a literature review underlines the main factors that influence cognitive effort. The methodology adopted towards the formalisation of cognitive effort is presented in section 3 . In section 4 we present our heuristic formalism built on these factors. We consider possible fields of applications in 5 and a synthesis of open issues and future works in section 6 . 


\section{Related Work and Review of Cognitive Effort}

Cognitive effort is a subjective phenomenon. One of the classic dilemmas of psychology concerns the division of attention among concurrent streams of mental activity. Humans often perform several activities in parallel. They suppress or queue stimuli on their behaviour organisation, underling an internal bottleneck characteristic on processing stimuli which can only operate on one stimulus or one response at a time 6 . Attention theories propose that the central neural system is limited, so humans are unable to think, remember, perceive or decide more than one thing at a time. Capacity theory provides a contrary view, assuming the existence of structural bottlenecks that supposes a limited humans' capacity allocable among concurrent activities 12. The concept of short-memory is introduced, that refers to the capacity of holding a small amount of information in mind in an active, readily available state for a short period of time. The more one acquires experience the less cognitive effort he consumes to resolve the same task. If the amount of cognitive effort that individuals allocate to a task decreases, as they become more skilled, the rate of change in cognitive effort should depend on the rate of skill acquisition 13. Long-term memory is the store of experience and results of skill acquisition. Arousal is an important factor in regulating attention because it is crucial for motivating certain behaviours 6. Arousal is a physiological and psychological concept which refers to the state of being awake. Motivation, perceived difficulty, subjective experience, psychological stress are all example of factors that play a role in directing attention towards a certain task. For instance, anxiety or boredom may have impact on performing certain activities. High ability individuals have larger pool of cognitive resources than low ability individuals who need to make larger resource adjustments to achieve the same outcome. Self-regulation theories 14, 15 suggest that individuals with different levels of cognitive ability may react to changes in task difficulty in different ways. Low ability people with a high degree of perceived difficulty require

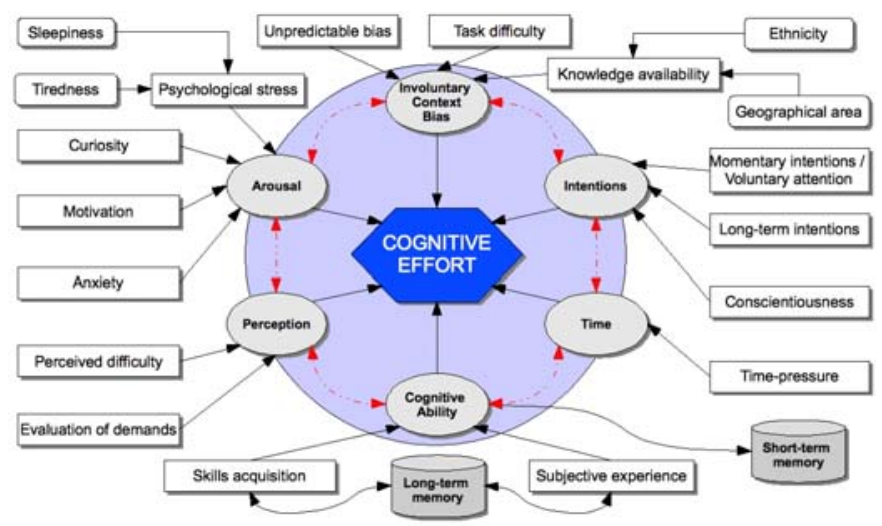

Fig. 1. Cognitive Effort influencing factors 
more cognitive effort in performing a task [16. The conscientiousness may moderate the level of attention on a task: highly conscientious individuals choose to work harder and persevere longer than individuals with lower level of conscientiousness 17]. We propose a summary of the main influencing factors in figure 1

\section{A Proposal Methodology}

One of the main difficulties in discussing cognitive effort is that the phenomenon has a subjective nature. Studies in psychology and neuro-sciences have attempted to provide a detailed definition of the concept but they demonstrated restricted scope over limited aspects of the concept. The present study, on the contrary, differs from the previous ones because we adopt an approach based on synthesis, that seeks to develop a more comprehensive basis for computing cognitive effort. For any definition of cognitive effort we can formulate a test of the formalism to understand whether it is suitable to the accepted definition. This approach is powerful because it may be seen as a refining stage towards a formalism that satisfies most people's views of cognitive effort, what it is and how it works. The formalism is conceived from experience, intuitive expectations about cognitive effort from a subjective point of view, and conclusions to be found in the psychological, philosophical and sociological literature. The methodology's goal is to merge together different observations, intuitions and definitions to build a simple formalism that is supposed to model the way cognitive effort behaves. We make no affirmations about the validity of the formalism, even if based on literature's studies, but we claim that the results of the application of the formalism to a consideration of cognitive effort is the same as if we had been considering the problem using real cognitive effort.

To deal with the formalisation of cognitive effort we propose to adopt the Popper methodology, presented in The Logic of Scientific Discovery 10 where he asserted that: a scientific theory should be based on a "falsification" approach in which no number of experiments can ever prove a theory but a single experiment can contradict one. He suggested that empirical theories are characterised by falsifiability and must satisfy the following criteria:

- demarcation: the theory must demarcate the area from pseudo-science, it must be testable, refutable and falsifiable [11.

- simplicity: the theory must be simple. Simplicity is better than complexity because it allows extreme tests and experimentations on the theory, making it more scientific than complex theories.

- replication/duplication: the theory must be capable of replication and/or duplication. Obtained results must be able to be repeated and as a consequence we can convince ourselves that we are not dealing with a mere isolated coincidence but with regular and reproducible events which are inter-subjectively testable.

Scientific theories are not static and they change perpetually: the formalism presented here can be continually refined. 


\section{An Example Heuristic Formalism}

Few studies have tried to measure cognitive effort and they can be classified in three groups [18: subjective or self-report measurement, performance and physiological measures. Self-report measures have always attracted researchers: no one is able to provide a more accurate judgement with respect to experienced mental load than the person concerned. However, self-report measures suffer from different rating scales and personal judgements. Performance measures are taskdependent and primary and secondary task approaches have been widely used so far, producing good results 18. Unfortunately, these techniques require laboratory tools to measure, for instance, the reaction-time useful to assess the amount of cognitive effort required for completing a task. Physiological measures represent the most accurate way of assessing mental workload, often unobtrusively, but they need appropriate equipment to measures physiological behaviours such as blood temperature, pupils dilation. The formalisation of cognitive effort as a computational concept needs to rely less on these classical measurements and it needs to focus on more general concepts easy to model. Indeed, we need a tool to monitor users' behaviour while performing a task, and we assume that this tool can be build up as a piece of software. We have analysed the factors involved in assessing cognitive effort and we propose a possible formalisation of each of them towards a general model.

Cognitive Ability: Some people obviously and consistently understand new concepts more quickly, solve new problem faster, see relationships and are more knowledgeable about a wider range of topics than others. Modern psychological theory views cognitive ability as a multidimensional concept and several studies, today known as IQ tests, tried to measure this trait 19. Carroll suggested [20] that there is a tendency for people who perform well in a specific range of activities, to perform well in all others as well. Recent work 21 suggests that some aspects of people's cognitive ability peak around the age of 22 and begin a slow decline starting around age 27 . However, as it is noted, there is a great deal of variance among people and most cognitive functions are at a highly effective level into their final years, even when living a long life. Some type of mental flexibility decreases relatively early in adulthood, but that how much knowledge one has, and the effectiveness of integrating it with one's abilities, may increase throughout all of adulthood if there are no pathological diseases. These considerations represent pieces of evidence that allow us to model cognitive ability with a growing function, i.e. a curve that starts at low levels and increases quickly to a growing rate threshold from which it still increases but moderately. The flexible sigmoid function proposed by Yin et al. in $\underline{22}$ is suitable for our purposes.

$$
\begin{gathered}
C A:\left[1 . . G_{t h}\right]^{3} \in \aleph^{3} \rightarrow[0 . .1] \in \Re \\
C A\left(G_{t h}, G_{r}, t\right)=C A_{\max }\left(1+\frac{G_{t h}-t}{G_{t h}-G_{r}}\right)\left(\frac{t}{G_{t h}}\right)^{\frac{G_{t h}}{G_{t h}-G_{r}}}
\end{gathered}
$$


where CA is the level of cognitive ability, $G_{t h}$ is the growing threshold, which we may set to an average of mortality of 85 years. $G_{r}$ is the growing rate, which we may set to 22 years, i.e. the point where the curve reaches the maximum growing weight and from that, increases moderately. $t$ is the age in years of a person and $C A_{\max }$ is the maximum level of cognitive ability an individual can reach, in this case equal to 1 . The properties $G_{t h}$ and $G_{r}$ are flexible because they can be chosen by considering environmental factors such as the degree of mortality or level of education of a country. For instance, if we consider a person 40 years old, with a growing rate of 22 years and a growing threshold of 85 years, by applying the formula above, we obtain 0.62 of cognitive ability.

Arousal: The concept of arousal was sometimes treated in literature as a unitary dimension, as if a subject's arousal state could be completely specified by a single measurement such as the size of his pupil 6. However this is a oversimplification and arousal is a multidimensional concept that may vary in different situations and, above all, there are several kinds of individual-subjective factors to consider. This is a relevant problem in studying subjective cognitive effort, but the main goal of our contribution is to present the main factors that influence it. For this reason, we propose a simple subjective arousal taxonomy where different type of arousal, such as curiosity, motivation, anxiety, psychological stress, are organised in a multi-level tree. In other words this represents a map of an individual subjective status before performing a certain task. A subjective arousal taxonomy is a 3 -tuple $\langle A, W, R\rangle$ composed by a set of pieces of arousal factors $A$ organised in a tree where their unidirectional relationship in the tree is defined in $\mathrm{R}$ by using the weights in W. Each node has at most one parent, except the root node which has no parent. Each internal node $a_{i}$ has a fixed influence strength $w_{i}$ towards his only parent. Just leaf nodes (node without children), with cardinality $\operatorname{Card}_{l n}$, have a value in $[0 . .1] \in \Re$ which indicates the degree of arousal (eg. 0 is not motivated at all, 1 is highly motivated) while each internal node's and the root node's values are inferred by the relationship with their children along with the related strength. The root of the tree is the final level of arousal that influences the degree of cognitive effort.

$$
\begin{gathered}
A:\left\{a_{1}, a_{2}, \ldots ., a_{n} \mid a_{i}:[0 . .1] \in \Re, 1 \leq i \leq n\right\} \\
W:\left\{w_{1}, w_{2}, \ldots ., w_{n} \mid w_{i}:[0 . .1] \in \Re, 1 \leq i \leq n\right\} \\
R:\left\{\forall a_{i} \exists ! r_{i} \mid r_{i}: A \times A \rightarrow W, r_{i}\left(a_{i}, a_{p}\right)=w_{i}\right\}
\end{gathered}
$$

where $a_{p}$ is $a_{i}$ 's parent. The degree of each arousal $a_{i}$ for leaf nodes is an explicit input value while the degree of each arousal $a_{i}$ for internal nodes is the weighted sum of its $c$ children's values.

$$
a_{i}^{i n t}=\left(\sum_{z=0}^{c}\left(a_{z} \cdot w_{z}\right)\right) \leq 1
$$

Finally the root's weight $w_{\text {root }}$ is 1 and, as it has no parent, its relation $r_{\text {root }}=\emptyset$. The root node inferred by applying the previous steps is:

$$
A_{\text {root }}: A^{\text {Card }_{l n}} \times W^{n} \times R^{n} \rightarrow[0 . .1] \in \Re
$$


Intentions: Subject's intentions have an important role in determining the level of cognitive effort while performing a task. This individual subjective concept may be splitted into short-term and long-term intentions. We propose to model these concepts with real values computing their average. We refer to short-term intentions or momentary intentions with $I_{s t}$ and to long-term intentions with $I_{l t}$ which are subjective judgements in the range [0..1] (0: no intentions at all, 1 : highly intentioned). Intentional shades can be modelled: a person can be highly intentioned to get a degree (long-term) but in short-time does not like examinations.

$$
\begin{gathered}
I:[0 . .1]^{2} \in \Re^{2} \rightarrow[0 . .1] \in \Re, I_{S T}:[0 . .1] \in \Re, I_{L T}[0 . .1] \in \Re \\
I\left(I_{S T}, I_{L T}\right)=\frac{I_{S T}+I_{L T}}{2}
\end{gathered}
$$

Involuntary Context Bias: Several factors can influence cognitive effort as pseudo-static and unpredictable biases. The latter refer to biases which are almost static and depend on environmental aspects. For instance, there is a large difference across ethnic groups and geographic areas in the available knowledge: people living in Africa have a reduced access on knowledge compared to people living in occidental countries so they may find a question more or less difficult. Similarly, another pseudo-static bias is the task difficulty. Even though it is hard to exactly estimate the complexity of different tasks, it is not expensive to claim that reading a newspaper demands less cognitive effort than resolving a math equation. Cognitive effort may be eventually influenced by unpredictable context biases. For instance, in a working context, phone ringing, questions from colleagues, e-mail delivering all represent involuntary context biases. We propose to use a ranking system to build up a task-difficulty dictionary and real values to model contextual available knowledge and unpredictable bias.

$$
\begin{gathered}
C B:[0 . .1] \in \Re^{3} \rightarrow[0 . .1] \in \Re^{3}, \quad C_{\text {know }}, T_{\text {diff }}, U_{\text {bias }}:[0 . .1] \in \Re \\
C B\left(C_{\text {know }}, T_{\text {diff } f}, U_{\text {bias }}\right)=\frac{1}{3} \cdot C_{\text {know }}+\frac{1}{3} \cdot T_{\text {diff }}+\frac{1}{3} \cdot U_{\text {bias }}
\end{gathered}
$$

where $C B$ is the total context bias, $C_{\text {know }}$ is the contextual knowledge availability, $T_{\text {diff }}$ is the task difficulty and $U_{\text {bias }}$ is the unpredictable bias. This formula is flexible because provides a way to model particular situations. We may formalise a situation where a person in Central Africa can not use the Internet, so low level of knowledge availability, performing an hard physics task in a noisy library.

Perception: The same task may be perceived differently by two subjects. Perceived difficulty is higher when individuals are presented to new tasks: they may not know what the optimal amount of effort is, given a particular difficulty level. We propose to model this concept as a value $P D:[0 . .1] \in \Re$ where values near 0 indicate tasks perceived to be very easy and values tending to 1 represent tasks perceived highly complex.

Time: Time is a crucial factor that must be considered in modelling cognitive effort. Time-pressure is sometimes imposed by explicit instruction to hurry and sometimes by demand characteristics of the task. In the former case a real value 
is sufficient to model the concept, while in the latter we may easily add a taskrelated time-pressure value to the task-difficulty dictionary previously proposed. Formally: $T_{\text {press }}:[0 . .1] \in \Re$. Furthermore, time is essential because performing a task is not a single-instant action, rather is an action over time. This fact suggests that the described factors that influence the level of cognitive effort need to be considered within an interval of time. Several temporal formal methods and theories are available in literature and studying the temporal-related aspect of cognitive effort requires a separate contribution. We remind this investigation to future work and in this first attempt we propose a simple cognitive effort formalism that propagates all the proposed factors at the same level over time. We assume the existence of a function $F_{\text {time }}: \aleph \rightarrow[0 . .1] \in \Re$ that models the trajectory of focused attention for each task over time: it returns the level of attention at a given time. Finally, we propose to model cognitive effort as a discrete function:

$$
\begin{gathered}
C E:\left([0 . .1]^{6} \in \Re^{6}\right) \times(\Re) \times(f: \aleph \rightarrow[0 . .1] \in \Re) \rightarrow \Re \\
C A^{\prime}=C A\left(G_{t h}, G_{r}, t\right), A^{\prime}=A_{\text {root }}, I^{\prime}=I\left(I_{\text {st }}, I_{l t}\right), \\
C B^{\prime}=C B\left(C_{\text {know }}, T_{\text {diff }}, U_{\text {bias }}\right), P D^{\prime}=P D, t_{p}^{\prime}=t_{\text {press }}, t^{\prime}=t \\
C E\left(C A^{\prime}, A^{\prime}, I^{\prime}, C B^{\prime}, P D^{\prime}, t_{p}^{\prime}, t^{\prime}\right)= \\
\sum_{i=0}^{t^{\prime}} F_{\text {time }}(i) \cdot \frac{\left[C A^{\prime}+A^{\prime}+I^{\prime}+C B^{\prime}+P D^{\prime}+t_{p}^{\prime}\right]}{6}
\end{gathered}
$$

where CA is cognitive ability, $A_{\text {root }}$ is arousals, I is intentions, CB is contextual bias, $t_{\text {press }}$ is time pressure, $f_{\text {time }}$ is the function for attention over time and $\mathrm{t}$ is the effective time spent to complete a task. Therefore, the final level of cognitive effort elicited on a task is a function of time and of the individual subjective status along with environmental properties.

\section{Possible Applications}

The formalism proposed in this paper may be applied in several disciplines such as computer science, psychology, neuro-science, economy. Here we present some example of its application. We assume all the cognitive effort influencing factors are available and can be gathered by using unobtrusive appropriate tools, monitors/loggers or derived from works and studies in literature. In education contexts, we may use the formalism to monitor the learning rate of students based on the hypothesis that students should show less cognitive effort in performing similar tasks due to their skill acquisition level. The more they acquire knowledge, the less cognitive effort they should spend in similar activities. Recommender systems may benefit from using the cognitive effort formalism, such as the online encyclopaedia Wikipedia, that foresees interactions among users and web-pages. The more users spend cognitive effort in contributing towards the improvement of an article, the more their contribution may be considered qualitative. Therefore, we may hypothesise that the trustworthiness of a Wikipedia 
article may depend on the quality of contributions. Similarly, in social search, assuming the existence of a logger that captures Internet users' behaviour while surfing web-pages, cognitive effort may be adopted to predict users' interests on a particular web document. Here the hypothesis is that the more users show a positive degree of cognitive effort on a web-page, the more that web-page may be considered interesting. Yet, if most of the users show similar level of cognitive effort on a web page, that means similar behaviours, we may infer a level of trustworthiness to it, which can be either positive or negative. Extending this concept to the WWW, a social search engine may be conceived. In online communities, such as blogs, forums, social networks, people interact with each other leaving feedback. Here the amount of cognitive effort may help to classify most active and trustworthy users: finance forum may benefit from our formalism. Measuring cognitive effort may be helpful for clinical purposes as well. If we can assess a degree of cognitive effort spent on a certain task, and we are able to do this repeatedly over time, we may predict people's addiction to that task. This is based on the hypothesis that addicted people show persistence of cognitive effort, that means the same behaviour on a task over time. A clinical addiction predictor may be adopted to have a first insight into the degree of addictiveness of online game player or betters. Similarly, psychologists may use the formalism as a preliminary tool to study individuals' addictiveness. In neuro-science, the application of the formalism may avoid the use of fMRI scanners for patients who show low addicted behaviour.

\section{Open Issues and Future Works}

This contribution is an introduction of a formalism for cognitive effort which is useful in clarifying and motivating discussion of the concept and is extensible to take into consideration further studies in the area. In addition the formalism is implementable, it offers the basis for the first implementation of cognitive effort in an intelligent artificial agent. Being based on simple mathematics, it provides the ideal tool for artificial agents in making reasoned decisions. The main aim of this contribution is to increase the understanding of cognitive effort and to provide a tool of great importance as an indicator of work which could be done. Despite these considerations and the intrinsic complexity of the phenomenon, cognitive effort is also to a large extent automatic, unconscious so further studies need to be carried out. Subjective cognitive effort, in our opinion, is a non-monotonic concept, further influencing factors may be added to the formalism attacking or supporting previous ones, other may be grouped, other ones deleted. A defeasible reasoning logic may describe the relations among factors. The phenomenon may be modelled by a more appropriate algebra or first-order logic. Several decisions contributed in the formalisation of cognitive effort: some of these imposed a general structure of cognitive effort which may be not always valid. This contribution is the first attempt in formalising cognitive effort as a computational concept, so it does not aim to be the final implementation but, instead, a first basic clarification tool that need to be faced and refined over 
time. It is beneficial to social science because it allows the precise discussion of the concept of cognitive effort. In the new Distributed Artificial Intelligence field it allows robustness and sensible behaviour in unpredictable and patchy environments and it allows agents to reason sensibly about other agents, either human or artificial.

\section{References}

1. Titchener, E.B.: Lectures on the Elementary Psychology of Feeling and Attention, p. 173. Macmillian, New York

2. Locke, E.A.: Toward a Theory of Task Motivation and Incentives. Organizational Behavior and Human Decision Processes 3(2) (1968)

3. Vroom, V.H.: Work and Motivation. Wiley, New York (1964)

4. Fried, Y., Slowik, L.H.: Enriching goal-setting theory with time: An Integrated Approach. Academy Management Review 29(3), 404-422

5. Berlyne, D.E.: Conflict, Arousal and Curiosity. McGraw-Hill, New York (1960)

6. Kahneman, D.: Attention and Effort. Prentice Hall, NJ (1973)

7. Norman, D.A., Bobrow, D.B.: On Data-limited and Resource-limited Processes. Cognitive Psychology 7, 44-64 (1975)

8. Humphreys, M.S., Revelle, W.: Personality, Motivation and Performance: A Theory of the Relationship Between Individual Differences and Information Processing. Psychological Review 91(2) (1984)

9. Schvaneveldt, R.W., Reid, G.B., Gomez, R.L., Rice, S.: Modeling mental workload. Cognitive Technology, 3, 9-31 (March 1998)

10. Popper, K.R.: The Logic of Scientific Discovery. Hutchinson (1967)

11. Popper, K.R.: Conjectures and Refutations. Routledge, New York (1969)

12. Moray, N., O'Brien, T.: Signal-detection theory applied to selective listening. Journal of the Acoustical Society of America 42, 765-772

13. Deadrick, D.L., Bennet, N., Russel, C.J.: Using HLM to Examine Dynamic, Performance Criteria Over Time. Journal Management 23(6)

14. Carver, C.S., Scheiner, M.F.: On the Self-regulation of Behavior. Cambridge University Press, Cambridge

15. Karoly, P.: Mechanisms of Self-regulation: A Systems View. Annual Review of Psychology 44, 23-52

16. Yeo, G., Neal, A.: Subjective Cognitive Effort: a Model of States, Traits, and Time. Journal of Applied Psychology 93(3), 617-631 (2008)

17. Barrick, M.R., Mount, M.K., Strauss, J.P.: Conscientiousness and Performance of Sales Representatives: Test of the Mediating Effects of Goal Setting. Journal of Applied Psychology 78(5), 715-722

18. O’ Donnell, R.D., Eggemeier, F.T.: Workload Assessment Methodology. Cognitive Processes \& Performance II, 1-49 (1986)

19. Dickens, T.W.: Cognitive Ability. In: Durlauf, S. (ed.) The New Palgrave Dictionary of Economics (forthcoming)

20. Carrroll, J.B.: Human Cognitive Abilities: A Survey of Factor-analytic Studies. Cambridge University Press, UK (1993)

21. Salthouse, T.: When does age-related cognitive decline begin? Journal of Neurobiology of Aging 30(4), 507-514 (2009)

22. Yin, X., Goudriaan, J., Lantinga, E.A., Vos, J., Spiertz, H.J.: A Flexible Sigmoid Function of Determinate Growth. Annals of Botany 91 (2003) 